Voix plurielles 9.2 (2012)

\title{
Le créole haïtien et les créoles des Petites Antilles et de la Guyane Quelques points de comparaison
}

Robert Damoiseau, Professeur émérite, Université des Antilles et de la Guyane

La proximité entre le créole haïtien et les créoles de la Martinique, de la Guadeloupe et de la Guyane permet l'intercompréhension dans l'espace américanocaraïbe partagé par les communautés concernées. Nous nous proposons d'examiner en quoi certaines des procédures mises en œuvre par ces créoles pour exploiter un fonds lexical commun, essentiellement d'origine française, présentent des analogies mais révèlent également des spécificités. L'analyse portera sur le syntagme nominal, les schèmes prédicatifs, et les systèmes aspecto-temporels et modaux.

\section{Le nom et les déterminants}

Les éléments susceptibles de remplir certaines fonctions (sujet, objet, circonstant..) et compatibles avec un certain nombre d'unités spécifiques (les « déterminants ») constituent, dans chacun des quatre créoles étudiés, la classe nominale. Les systèmes de détermination des noyaux nominaux dans ces créoles s'organisent selon des règles qui montrent de fortes analogies.

Pour ce qui est du déterminant, connaissant la fréquence d'emploi la plus élevée dans chacun des systèmes, le déterminant «spécifique», on notera d'abord son positionnement après le nom. En relation avec sa postposition au nom qu'il détermine, cet élément connaît, dans trois ${ }^{1}$ de ces quatre créoles, des variations formelles conditionnées par la finale du nom.

Sur ce point particulier, les créoles haïtien et martiniquais sont très proches. Le déterminant présente en effet une initiale consonantique lorsque la finale du nom est une consonne ou une semi-consonne :

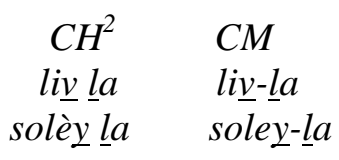

Si la finale est une voyelle, le déterminant présente une forme vocalique :

$\begin{array}{ll}\text { CH } & C M \\ \text { gato } \underline{a} & \text { gato } \underline{a} \underline{a}\end{array}$


Ces deux créoles se distinguent du créole guyanais, dans lequel la forme du déterminant est toujours de type vocalique, quelle que soit la finale du nom déterminé :

CGuy

liv $\underline{-} \underline{a}$

soley- $\underline{a}$

gato- $\underline{a}$

Cependant, en guyanais comme en haïtien ${ }^{3}$ et en martiniquais joue le phénomène de nasalisation progressive, du nom vers le déterminant :

\begin{tabular}{lll}
$C H$ & $C M$ & \multicolumn{2}{c}{ CGuy } \\
moun $\underline{\text { nan }}$ & moun-lan & moun-an \\
koch $\underline{\text { on } \underline{\text { an }}}$ & kochon- $\underline{\text { an }}$ & kochon- $\underline{\text { an }}$
\end{tabular}

On notera que pour ce qui est du haïtien, dans le cas d'une consonne nasale en position finale $\mathrm{du}$ nom, la nasalisation affecte non seulement la voyelle $\mathrm{du}$ déterminant, mais également la consonne (moun nan).

Pour ce qui est de la valeur de ce déterminant, il convient de souligner qu'il fonctionne, prioritairement, dans les quatre créoles en tant que déictique, c'est-à-dire comme instrument d'ancrage du référent nominal à la situation d'énonciation. Dans une moindre mesure, il peut servir à désigner un référent déjà connu des interlocuteurs (cf emploi «anaphorique»). Mais sa valeur spécifiante est forte, en tout cas plus prononcée que celle du déterminant «défini» du français: en conséquence - et contrairement à ce qu'on constate pour le «défini » en français - son emploi est incompatible avec l'emploi «générique » du nom, qui repose sur une opération de désancrage de l'énoncé par rapport à la situation d'énonciation.

-Le créole haïtien, comme les trois autres créoles considérés, dispose d'un déterminant démonstratif qui permet de viser un référent dans l'environnement immédiat des participants à l'acte d'énonciation et donc de le sélectionner par rapport à d'autres référents. En relation avec sa force déictique plus accentuée que le spécifique, son signifiant révèle un renforcement de ce déterminant (par sa en $\mathrm{CH}$, CGua et CGuy, par ta en $C M$ ). Mais le positionnement de cet élément de renforcement par rapport au déterminant spécifique et par rapport au nom déterminé varie selon les créoles : 
Voix plurielles $9.2(2012)$

-il précède le spécifique en $C H$ et en $C M$ :

$\begin{array}{ll}\mathrm{CH} & \mathrm{CM} \\ \text { moun } \underline{\text { sa }} \text { a } & \text { moun- } \underline{\text { tala }}(\underline{\text { taa }})\end{array}$

-il lui est postposé en $C G u a$ :

CGua

moun-lasa

-il est antéposé au groupe : Nom+dét. spécifique en CGuy:

CGuy

sa moun-an

-Une règle commune aux quatre créoles concerne la pluralisation : cette opération ne s'exerce en effet que sur des référents nominaux préalablement déterminés par le spécifique ou le démonstratif. Cette exigence révèle que la vision pluralisatrice, dans ces langues, implique que les référents soient préalablement clairement repérés en tant qu'entités, donc identifiés comme éléments comptables.

Cependant, les quatre créoles n'ont pas retenu les mêmes procédures d'expression du nombre.

-Les créoles martiniquais et guadeloupéen recourent au morphème sé, antéposé au syntagme N+déterminant, pour marquer le pluriel :

$\begin{array}{cc}C M & \text { CGua } \\ \text { moun-lan } \rightarrow \underline{\text { sé }} \text { moun-lan } & \text { moun-la } \rightarrow \underline{\text { sé moun-la }} \\ \text { moun-tala } \rightarrow \underline{\text { sé }} \text { moun-tala } & \text { moun-lasa } \rightarrow \underline{\text { sé moun-lasa }}\end{array}$

-En créole haïtien et en créole guyanais, c'est le morphème de personne : yo en $C H$, yé en $C G u y$ (cf : ils/elles en français) qui, postposé au nom, marque le pluriel ${ }^{4}$.

En guyanais, il se produit une élision de la voyelle finale du morphème yé au contact du déterminant $a$ ou an :

$$
\begin{gathered}
\text { CGuy } \\
l i v-a \rightarrow l i v \text { yé } a \rightarrow l i v-y a \\
\text { moun-an } \rightarrow \text { liv yé } a n \rightarrow l i v-y a n
\end{gathered}
$$

En haïtien, le principe de liaison entre l'opération de pluralisation et celle de détermination est exploité de façon optimale : la pluralisation impliquant la détermination, la seule marque yo est porteuse à la fois de l'information «pluriel » et de l'information « spécifique ». 
$\mathrm{CH}$

liv la $\rightarrow$ liv $\underline{\text { yo }}$

moun nan $\rightarrow$ moun yo

On a affaire là à un phénomène d'amalgame ${ }^{5}$ au sens fonctionnaliste du terme : au seul signifiant de $\underline{y o}$ correspondent deux signifiés, à savoir «pluriel » et « spécifique ».

-L'expression de la possession met directement en jeu les morphèmes de personne dans les créoles haïtien, martiniquais et guadeloupéen. Le guyanais se distingue de ces créoles dans la mesure où il possède des unités spécialisées dans l'expression de la possession, qu'il antépose au nom : $\underline{\text { mo }}$ frè, $\underline{\text { to }} . . ., \underline{\text { so }} . . ., \underline{\text { nou }} . .$. , $\underline{z o t} \ldots, \underline{y e ́} . .$.

Dans les trois autres créoles, c'est le morphème de personne, fonctionnant comme complément de nom, qui exprime la possession :

\section{CH CM CGua \\ frè $\underline{\text { mwen }}$ frè- $\underline{\text { mwen }}$ frè an $\underline{\text { mwen }}$}

Comme tout complément de nom, le morphème de personne est directement postposé au nom en haïtien et en martiniquais, alors le guadeloupéen fait intervenir la préposition $\underline{a}$, qui se nasalise en relation avec la présence de consonnes ou de voyelles nasales présentées par le signifiant du morphème de personne :

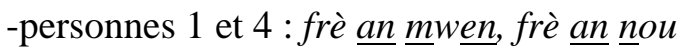

-personnes 2, 3, 5 et $6:$ frè $\underline{a}$ 'w, frè $\underline{a}$ 'y, frè a zot, frè $\underline{a}$ yo.

La singularité du créole haïtien tient à ce qu'il présente un système de morphèmes de personne qui comporte 5 unités alors que ceux des trois autres créoles en comportent 6. Là où le martiniquais, le guadeloupéen et le guyanais ont une forme de personne 4 (nou), et une de personne 5 (zot), l'haïtien dispose d'une forme, nou, qui, en fonction de la situation d'énonciation, correspond soit à la personne 4 (cf. nou), soit à la personne 5 (cf. zot) dans les autres systèmes. Ainsi pour les morphèmes de personne : 
Voix plurielles $9.2(2012)$

\begin{tabular}{|c|c|c|c|}
\hline CH & CM & CGua & CGuy \\
\hline mwen & mwen & mwen & mo \\
\hline \multirow{2}{*}{ ou } & ou & ou & to \\
\hline \multirow{2}{*}{ li } & $i$ & $i$ & $i$ \\
\hline \multirow{2}{*}{ nou } & nou & nou & nou \\
\cline { 2 - 4 } & zot & $z$ zot & $z$ ot \\
\hline yo & $y o$ & $y o$ & $y e ́$ \\
\hline
\end{tabular}

L'économie que représente le fonctionnement du système haïtien repose sur le fait qu'il exploite de façon optimale les conditions pragmatiques de l'échange communicatif. Ce qui est en jeu ici, c'est l'inclusion de l'énonciateur dans la valeur référentielle de nou (pers. 4, nou, dans les autres systèmes) versus son exclusion (pers. 5, zot dans les autres systèmes). Le degré d'implication de l'énonciateur dans la relation à l'allocutaire l'inclut ou non dans le référent de nou ${ }^{6}$.

On notera enfin que le lien entre pluralisation et détermination du nom apparaît clairement dans la structure exprimant la possession dans les quatre créoles.

Dans les créoles martiniquais, guadeloupéen et guyanais, la pluralisation du syntagme :

$\begin{array}{ccc}C M & \text { CGua CGuy } \\ \text { frè-mwen } & \text { frè an mwen }\end{array}$

implique la determination par le spécifique :

CM CGua CGuy

$\rightarrow$ séfrè mwen- $\underline{a n} \quad \rightarrow$ séfrè mwen-la $\quad \underline{\text { mo frè-yan }}$

En créole haïtien, les deux informations «spécifique» et «pluriel»sont apportées par yo (cf supra) :

\section{$\mathrm{CH}$}

frè mwen $\rightarrow$ frè mwen $\underline{\text { yo }}$

-L'expression de la circonstance, et notamment la localisation de la relation prédicative s'effectue fréquemment par un nom précédé d'une préposition : 
CH M wè tifi a nan lari a

CM Man wè tifi-a an lari-a

CGua An wè tifi-la an lari-la

CGuy Mo wè tifi-a annan lari-a

J'ai vu la jeune fille dans la rue

Cependant, chaque créole dispose d'un stock d'unités nominales référant à des lieux pouvant fonctionner comme circonstants sans recours à la préposition. Ces lieux sont assez familiers aux membres de chacune des communautés créolophones pour que les noms qui y réfèrent, puissent être employés, comme des noms propres, sans déterminant et sans préposition :

\section{CH M wè tifi a legliz}

CM Man wè tifi-a légliz

CGua An wè tifi-la légliz

CGuy Mo wè tifi-a légliz

J'ai vu la jeune fille à l'église

Ce qui autorise, pour chaque créole, ce type de fonctionnement, est étroitement lié à la valeur de repère spatial, pour l'ensemble des membres de chaque communauté, des lieux auxquels réfèrent les noms en question. La comparaison révèle l'existence d'un fonds commun aux quatre créoles, mais également de spécificités en matière de "pertinence ${ }^{7}$ accordée par les communautés à tel ou tel nom, en fonction de données constitutives de leur environnement physico-culturel. Ainsi, si la valeur référentielle de lanmè (mer) l'autorise à fonctionner sans relateur dans les créoles des Antilles et de la Guyane, ce n'est pas le cas en créole haïtien, où le relateur est de mise :

\section{CM/CGua Yo alé lanmè \\ CGuy Yé alé lanmè \\ $\mathrm{CH} \quad$ Yo alé nan lanmè \\ Ils sont allés à la mer}

Ce point relatif à certains noms dans les créoles concernés livre des informations quant aux relations sémantico-syntaxiques dans le fonctionnement de la langue.

\section{Les schèmes prédicatifs}

Dans les quatre créoles, le rôle de noyau prédicatif est assuré avec la fréquence la plus élevée par un verbe. Avec les verbes bivalents, la structure de la phrase suit l'ordre SVO : 


\section{$\begin{array}{lll}S & V & O\end{array}$}

$\mathrm{CH} \quad$ Frè mwen gen yon machin

CM Frè-mwen ni an loto

CGua Frè an mwen ni on vwati

CGuy Mo frè gen roun loto

Mon frère a une voiture

Dans le cas de verbes trivalents, qui impliquent un transfert, les deux compléments sont construits directement, celui désignant le destinataire précédent qui correspond à l'objet de ce transfert :

CH Li bay timoun nan liv la

CM I ba timanmay-la liv-la

CGua I ba timoun-la liv-la

CGuy I bay timoun-an liv-a

Elle a donné le livre à l'enfant

-Lorsque le noyau prédicatif est un adjectif, le trait correspondant à «la propriété » porté par le signifié de l'adjectif est directement mis en rapport, dans les quatre créoles, avec le sujet :

\section{CH Dlo a cho \\ CM Dlo-a cho}

CGua Dlo-la cho

CGuy Dilo-a cho

L'eau est chaude

Il en va de même dans les cas où le prédicat est un élément de «localisation » (adverbe, groupe prépositionnel, nom qui réfère à un lieu connu) :

\begin{tabular}{llll} 
CH & Timoun yo la & Yo nan lakou a & Yo lekòl \\
CM & Sé timanmay-la la & Yo an lakou-a & Yo lékol \\
CGua Sé timoun-la la & Yo an lakou-la & Yo lékol \\
CGuy Timoun-yan la & Yé annan lakou-a & Yé lékol \\
\multicolumn{1}{c}{ Les enfants sont là } & Ils sont dans la cour & Ils sont à l'école
\end{tabular}

-Mais si le noyau prédicatif est un nom, la règle exige, dans les quatre créoles, la mise en œuvre de la copule (se en $C H$, sé en $C M$ et $C G u a$, sa en $C G u y$ ) :

$\mathrm{CH} \quad \mathrm{Ou}$ se yon timoun

CM Ou sé an timanmay

CGua Ou sé on timoun

CGuy To sa roun timoun

Tu es un enfant (et non un adulte)

En créole haïtien comme dans les autres créoles ici concernés, le signifié de certains noms les prédispose à la caractérisation du sujet. La langue exploite alors ce signifié non plus pour identifier le sujet en tant qu'entité constitutive d'un ensemble 
(cf énoncés précédents), mais pour le caractériser en lui affectant le trait définitoire de cette entité, qui devient alors une propriété. $\mathrm{Cf}$ :

\section{CH Ou timoun toujou \\ CM Ou timanmay toujou \\ CGua Ou timoun toujou \\ CGuy To timoun toujou}

Tu es resté enfant (tu as un comportement puéril)

On doit noter que l'affectation de cette charge signifiée à l'opération de caractérisation s'effectue en relation étroite avec les données qui structurent l'environnement et l'organisation socio-culturelle de chacune des communautés créolophones concernées. Ces corrélations apparaissent notamment de façon claire lorsqu'il s'agit d'affecter des noms référant à des animaux à la caractérisation d'humains. L'évaluation du degré de pertinence des comportements animaliers les autorisant à s'appliquer à des humains est le propre de chacune des communautés. C'est ainsi par exemple que les créoles haïtien et martiniquais ont retenu le « crabe sirik » comme représentatif de la pingrerie d'une personne, alors que le créole guyanais a confié ce rôle au « rat». Cf :

CH/CM Ou sirik

CGuy To rat

Tu es radin

Outre qu'ils révèlent «l'imbrication sémantico-syntaxique propre à un idiome » (François-Geiger 154), ces faits témoignent des analogies et des spécificités que présentent les créoles concernés dans leurs procédures d'appropriation du lexique qui leur a été légué, dans des conditions socio-économiques particulières, par le français.

\section{Les systèmes aspecto-temporels et modaux}

Pour ce qui est de l'expression de l'aspect, du temps et du mode, l'analyse comparative conduit à regrouper les créoles martiniquais, guadeloupéen et guyanais : on parle alors de «système en $\boldsymbol{k} \boldsymbol{a}$ ». Dans le cas du créole haïtien, les opérations qui correspondent à l'expression de ces notions conduisent à poser un système désigné comme « système en $a p$ ». 
Voix plurielles 9.2 (2012)

Les deux systèmes sont construits autour d'un axe aspectuel de type imperfectif-perfectif ${ }^{8}$. Cette opposition aspectuelle centrale s'exprime par les marques $\boldsymbol{k a} \boldsymbol{\emptyset} \emptyset$ en $C M$, CGua et $C G u y$, par les marques $\boldsymbol{a p}-\boldsymbol{\emptyset}$ en $C H$ :

$\begin{array}{lll}\text { CM/CGua/CGuy } & \text { Lapli ka tonbé } & \text { Lapli } \emptyset \text { tonbé } \\ \text { CH } & \text { Lapli ap tonbe } & \text { Lapli } \emptyset \text { tonbe } \\ & \text { Il pleut } & \text { Il a plu }\end{array}$

De façon symétrique dans les deux systèmes, l'expression de l'aspect sert de socle à celle du temps passé. La marque té/te se combine :

-d'une part à $k a$ et $a p$ :

\section{CM/CGua/CGuy Lapli té ka tonbé}

$\mathrm{CH} \quad$ Lapli t(e) ap tonbe

$\rightarrow$ Le déroulement du processus est situé dans le passé : cf «Il pleuvait »

-d'autre part à $\emptyset$ :

\section{CM/CGua/CGuy Lapli té Ø tonbé}

$\mathrm{CH} \quad$ Lapli te $\emptyset$ tonbe

$\rightarrow$ Le terme du processus est situé dans le passé : cf «Il avait plu».

-Les créoles martiniquais et guadeloupéen ${ }^{9}$ d'une part, le créole haïtien d'autre part, disposent d'une marque spécifique pour l'expression de l'aspect prospectif : $\boldsymbol{k a y}^{10}$ en $C M / C G u a$, pral (var. pray dans le Nord d'Haïti). Dans les deux cas, le recours au verbe de déplacement (ay en $C M / C G u a$ et $\mathrm{CH}$ Nord, al(e) en $\mathrm{CH}$, cf «aller» en français) permet d'exprimer la concomitance entre le début du déroulement de l'action ou du processus envisagé et le moment d'énonciation. L'effet de sens qui est lié à cet emploi permet au locuteur de présenter ce déroulement dans la continuité de la situation d'énonciation :

\section{CM/CGua Gadé! Lapli kay tonbé \\ $\mathrm{CH} \quad$ Gade! Lapli pral tonbe \\ Regarde! Il va pleuvoir}

C'est cette absence de décalage entre l'énoncé et le cadre physique qui le porte, qui conduit à interpréter la réalisation du processus évoqué comme imminente.

-Les deux systèmes présentent cependant une différence notable : elle tient au degré d'ancrage différent de l'axe imperfectif-perfectif à la situation d'énonciation.

L'emploi des deux marques $\boldsymbol{k} \boldsymbol{a}$ et $\boldsymbol{a p}$, dans chacun des deux systèmes, témoigne du souhait des locuteurs de présenter le déroulement d'un procès en liaison étroite avec le cadre dans lequel l'énoncé est émis. Ainsi, le contexte locatif de 
fonctionnement de $\boldsymbol{k} \boldsymbol{a}$ a souvent été évoqué par les analystes ${ }^{11}$ en relation avec sa valeur d'instrument de marque du progressif, c'est-à-dire du type d'aspect imperfectif correspondant à la présentation du déroulement d'un procès en coïncidence stricte avec le cadre « hic » et « nunc » de l'énonciation. Cf :

$C M$
I bò kay-la ka atann mwen
I la ka atann mwen
Il est près de la maison à m'attendre
Il est là à m'attendre

De même, on peut raisonnablement avancer que le signifié originellement spatial de «après », en français, à l'origine de son emploi au sein de la suite « être après à + infinitif », est à mettre en relation avec le fonctionnement de $a p$ et sa valeur de marque du progressif dans le système haïtien.

Mais il se trouve que, partant du progressif, le champ de fonctionnement de $\boldsymbol{k a}$ s'est ouvert à l'expression de l'itération, jusqu'à celle du général (d'où son emploi dans les proverbes). $\mathrm{Cf}$ :

\section{Jinet $\boldsymbol{k a}$ vini wè mwen toulédimanch Dlo pa ka monté mòn CGua ..................vwè \\ CGuy Jinet $\boldsymbol{k}$ a vini wè mo toulédimanch Dilo pa ka monté morn \\ Ginette vient me voir tous les dimanches L'eau ne grimpe pas les mornes (fig. : à l'impossible nul n'est tenu)}

Ce processus d'élargissement de la vision imperfective, qui va de pair avec la présentation d'un procès se déroulant hors du cadre physique de l'énonciation, n'est pas compatible avec le poids de la marque $\boldsymbol{a p}$ en tant qu'instrument du progressif dans le système haïtien. Son emploi suppose en effet que le procès évoqué se déroule en stricte coïncidence avec la situation d'énonciation. L'expression de l'itération est alors prise en charge par un auxiliaire spécifique :

\section{CH Jinet konn vini wè mwen chak dimanch}

Pour ce qui est de l'expression du général, qui correspond à une opération de désancrage total du procès par rapport à la situation d'énonciation, elle échappe, dans ce système, au champ aspectuel, et se caractérise par une absence de marquage, à l'instar des prédicats non processifs, qui, de par leur signifié, sont écartés de toute démarche de limitation :

\section{CH Dlo pa monté mòn}


En liaison avec la forte valeur de progressif de ap, le marquage des prédicats processifs par $\emptyset$ est celle d'un perfectif résultatif : quand il fait le choix de ne pas utiliser ap, le locuteur signifie non seulement que le procès nommé par le prédicat a atteint son terme, mais que ses effets sont constatables dans la situation d'énonciation. Ainsi, l'information apportée par un énoncé du type Lapli $\emptyset$ tonbe $(\mathrm{CH})$ est centrée sur les conséquences constatables « hic » et «nunc» de la réalisation du procès nommé par le prédicat.

Au plan de l'organisation du système, il faut également noter que la puissance de l'ancrage de la vision aspectuelle de type progressif-résultatif à la situation d'énonciation permet à la marque ap de prendre en charge l'expression du futur. En effet, dès lors que rien, dans l'environnement immédiat des interlocuteurs, n'atteste du déroulement en cours du procès, l'emploi de $\boldsymbol{a p}$ conduit à la lecture de ce déroulement comme étant «à venir ». C'est ainsi que l'énoncé : CH Lapli ap tonbe privé de sa validation expérientielle, va être interprété systématiquement comme référant à un événement futur (cf : «Il pleuvra »).

Ce fonctionnement de ap comme marque de temporalisation est lié au besoin qu'éprouve l'énonciateur d'assurer son interlocuteur de la réalisation d'un procès dont la réalité n'atteste pas le caractère effectif : privé de cet appui, il recourt, dans une démarche de compensation, à la forme même qui originellement est liée au constat « hic » et « nunc » de cette réalisation. Ce mouvement d'implication de l'énonciateur dans son assertion est la caractéristique même de la modalisation.

La langue affecte la valeur première de ap, qui est celle d'un instrument de validation de la réalisation d'un procès dans les limites de la situation d'énonciation, à la tâche qui consiste pour l'énonciateur à assurer l'allocutaire du déroulement de ce procès qui n'est pas attesté par la réalité : cette stratégie, conforme au principe de modalisation, produit un effet de temporalisation « futur ».

Cependant, en conformité avec la valeur testimoniale inhérente à son emploi premier en tant que marque de progressif, ap exprime un futur «certain», et se distingue en cela de $\boldsymbol{a}$ (ou $\boldsymbol{v a}$ ), qui exprime le futur «éventuel». Cf :

\section{CH M ap fè travay la kanmenm}

Je ferai le travail de toute façon
Ma fè travay la si m gen tan Je ferai le travail si j'ai le temps

En relation avec l'extension de son aire de fonctionnement jusqu'à l'expression du général, $\boldsymbol{k} \boldsymbol{a}$, au sein du système des Petites Antilles et de la Guyane, 
ne possède pas la force «implicative $»^{12}$ d'une stricte marque de progressif. De ce fait, il n'a pas la puissance modale qui caractérise ap dans le système haïtien. L'économie du système des Petites Antilles et de la Guyane a conduit à la mise en place d'une marque spécifique ${ }^{13}$ permettant la prise en charge par le locuteur de la réalisation d'un procès à venir: il s'agit de ké, instrument de l'expression de la temporalisation «futur» en martiniquais et guadeloupéen, mais servant soit l'expression du temps «futur », soit celle de l'aspect «prospectif » en guyanais, selon les liens entre l'énoncé et la situation d'énonciation (cf note 10).

CH M ap vini dimanch

CM Man ké vini dimanch

CGua An ké vin dimanch

CGuy Mo ké vini dimanch

Je viendrai dimanche

-Le parallélisme entre les deux systèmes se rétablit pour ce qui est de l'expression du conditionnel. Dans le système en $\boldsymbol{k} \boldsymbol{a}$ comme dans celui en $\boldsymbol{a p}$, le caractère hypothétique de la réalisation d'un procès s'exprime par la combinaison de la marque du futur ( $\boldsymbol{k} e$ en $C M / C G u a / C G u y, \boldsymbol{a}$ ou $\boldsymbol{a p}$ en $C H$ ) et de celle du passé (té/te) :

CM : Si mwen té ka travay, mwen té ké achté an loto CGua: Si mwen té ka travay, mwen té ké achté on vwati

CGuy : Si mo té ka travay, mo té ké achté roun loto

$\mathrm{CH}$ : Si mwen te travay, mwen ta/tap achte yon machin

Le choix de la marque du futur est conforme à la présentation du procès (ici l'achat) comme une projection de la part du locuteur, celui de la marque du passé signifie que cette projection est coupée de son $\operatorname{socle}^{14}$ que représente la situation d'énonciation, d'où le caractère « irréel » de ce procès.

La seule différence réside dans le fait qu'en $C M / C G u a / C G u y$, la possibilité de faire jouer l'opposition aspectuelle té $\boldsymbol{k a} \boldsymbol{a}$-té $\emptyset$, avec un prédicat processif, permet de présenter la condition exprimée dans la proposition subordonnée :

-comme réalisable : CM/CGua : Si mwen té ka travay, mwen té ké achté...

CGuy: Si mo té ka travay, mo té ké achté... Si je travaillais, j'achèterais...

-ou comme non réalisée: $\quad C M / C G u a$ : Si mwen té $\emptyset$ travay, mwen té ké achté...

CGuy : Si mo té Ø travay, mwen té ké achté... Si j'avais travaillé, j'aurais acheté... 
En relation avec cette information, la réalisation du procès exprimé par le prédicat de la proposition principale sera interprété comme envisageable (cf. le « conditionnel présent » en français) ou non (cf. le « conditionnel passé » en français).

Dans le système haïtien, la vision aspectuelle du déroulement d'un procès sous l'angle progressif-résultatif exprimée par $\boldsymbol{a p}-\emptyset$ n'est pas compatible avec sa présentation dans un cadre hypothétique fourni par la subordonnée conditionnelle introduite par si: ce sont les indices situationnels qui orientent l'allocutaire vers une interprétation de la suite $t a / t$ ap + prédicat de la principale comme exprimant un procès réalisable ou non réalisé (voir plus haut).

Cette étude, limitée à quelques points, apporte un éclairage sur certaines des procédures mises en œuvre par les communautés d'Haïti, des Antilles et de la Guyane, pour s'approprier un lexique français et répondre aux besoins communicatifs qui s'imposaient à elles. L'examen de ces procédures révèle une grande proximité, qui conduit à certains types de rapprochements et de regroupements entre les créoles concernés mais il témoigne également de l'existence de traits propres qui fondent l'originalité de chacun d'entre eux au sein de l'espace qu'ils partagent.

Ces réalités, d'un intérêt majeur en linguistique comparative, sont encore largement exclues des préoccupations des didacticiens : il y a là tout un domaine qui appelle des recherches appliquées à l'enseignement des langues créoles, mais également à celui du français, dont le positionnement si particulier par rapport à ces langues est encore trop souvent méconnu et inexploité.

\section{Références bibliographiques}

Alexandre, C., A. Bentolila et A. Fauchois. «Les modalités en créole haïtien: approche syntaxique et rhétorique ». Espace créole 5 (1983) : 125-202.

Bernabé, J. Précis de syntaxe créole. Ibis Rouge, PU Créoles/GEREC F, 2003.

Cohen, D. L'aspect verbal. Paris : P.U.F, 1989.

Damoiseau, R. Recherche sur les notions de temps et d'aspect en créole haïtien et en français. Publications du Centre de Linguistique Appliquée, Université d'Etat d'Haïti, 1989a.

---. «Situation de communication et fonctionnement de la langue en créole haïtien ». La Linguistique XIV, 1 (1989b) : 30-42. 
Voix plurielles 9.2 (2012)

---. (en collaboration avec G. Jean-Paul, Préface de Pierre Vernet). Ann'aprann pale kreyòl! Faculté de Linguistique Appliquée, Université d'Etat d'Haïti, Karthala, 2002.

---. Éléments de grammaire comparée français-créole haïtien. Ibis Rouge, PU Créoles/GEREC F, 2005.

---. « De la prédication seconde à la prédication première: $K A$ en créole martiniquais ». Recherches et Ressources en Education et Formation, Revue de l'IUFM de Guadeloupe 2 (2008) : 11-16.

---. Syntaxe créole comparée, (Martinique, Guadeloupe, Guyane, Haïti). Sceren, Karthala, 2012.

François-Geiger, D. A la recherche du sens. Peeters-SELAF, 1990.

Hagège, Cl. La structure des langues, Coll. Que sais-je ? Paris : P.U.F., 1982.

Manessy, G. «Remarques sur la pluralisation du nom en créole et dans les langues africaines ». Etudes Créoles 1-2 (1985) : 129-143.

Valdman, A. et P. Vernet. Enquête sur la forme du déterminant défini en créole haïtien., Faculté de Linguistique Appliquée, Université d'Etat d'Haïti, 1982.

Vernet, P. Techniques d'écriture du créole haïtien., Centre de Linguistique Appliquée, Université d'Etat d'Haïti, 1980.

---. Langues, éducation et société en Haït. Thèse de Doctorat, Paris V Sorbonne, 1984.

\footnotetext{
Notes

${ }^{1}$ Seul le créole guadeloupéen présente une forme qui reste stable, la, quelle que soit la finale du nom déterminé.

${ }^{2} \mathrm{CH}$ : créole haïtien, $C M$ : créole martiniquais, $C G u a$ : créole guadeloupéen, $C G u y$ : créole guyanais.

${ }^{3}$ Une recherche menée par Pierre Vernet et Albert Valdman met en évidence un phénomène de nasalisation du déterminant sans la présence de voyelle ou de consonne nasales au sein ou en finale du nom (ex : tab lan). Ce phénomène concernerait les locuteurs jeunes, représentatifs des classes aisées de la capitale.

${ }^{4}$ Selon Manessy, il s'agit d'une procédure à laquelle recourent d'autres langues, notamment le yoruba, ainsi que certains créoles à bases lexicales anglaise, hollandaise, portugaise, espagnole.

${ }^{5}$ Cf. Alexandre, Bentolila et Fauchois.

${ }^{6} \mathrm{Cf}$. sur ce point Damoiseau (1989).

${ }^{7}$ Claude Hagège note l'existence, dans de nombreuses langues, de «... noms de lieux qui, investis d'une pertinence écologique et professionnelle, sont assez bien homologués pour ne pas exiger de relateurs...» (119).

${ }^{8} \mathrm{La}$ vision aspectuelle de ce type exige des prédicats à signifié processif, donc référant à des actions ou des processus : ce sont, dans leur grande majorité, des prédicats verbaux. Les prédicats statifs (une minorité de verbes, qui n'expriment ni une action ni un processus, ainsi que les prédicats non verbaux) ont une compatibilité réduite avec les marques d'aspect. En revanche, leur signifié est totalement compatible avec la temporalisation et les marques qui l'expriment.
} 
${ }^{9}$ Le créole guyanais se distingue, sur ce point, du martiniquais et du guadeloupéen : il dispose d'une seule marque, $\boldsymbol{k} \boldsymbol{e}$, qui, en fonction de la situation d'énonciation, peut exprimer soit l'aspect prospectif (Gadé! Lapli ké tonbé), soit le temps futur (Lapli ké tonbé dimen).

${ }^{10}$ On relève les variantes $\boldsymbol{k} \grave{e} \boldsymbol{y}$ et kéy en $C M$.

${ }^{11}$ Sur la mise en rapport de la forme de $\boldsymbol{k} \boldsymbol{a}$ et la relative déictique, voir Damoiseau (2008).

12 David Cohen, selon lequel une des «...deux valeurs stylistiques du progressif...est une valeur implicative par laquelle le locuteur s'implique dans l'affirmation de (la) relation (du sujet avec le procès) en la présentant avec un relief particulier. » (131).

${ }^{13}$ Il subsiste, des traces, chez certains locuteurs âgés, en créole martiniquais, de l'emploi d'une marque $\boldsymbol{a}$, à mettre en rapport avec le morphème $\boldsymbol{a}$ du créole haïtien, pour l'expression du futur hypothétique.

${ }^{14}$ Cet emploi de télte se fonde sur sa valeur d'instrument fort de temporalisation «passé » dans chacun des deux systèmes : son utilisation correspond au souhait du locuteur de poser le procès en rupture par rapport à la situation d'énonciation. 